\title{
Holomorphic differentials on open Riemann surfaces
}

\author{
By \\ Y. Kusunoki and Y. SAINOUGH
}

(Received September 25, 1970)

\section{Introduction}

The present paper consists of two parts. In the first part we shall show the existence of an Abelian differential with given divisor and periods on any open Riemann surface. This can be regarded as a generalization of the Gunning-Narasimhan's theorem [3] which states that on any open Riemann surface there exists a holomorphic exact differential without zeros. The proof can be achieved by a slight modification of their ingenious method. As a result we know that for every Abelian differential $\omega$ there exists a holomorphic function $f(\neq 0)$ such that a differential $f \omega$ possesses the prescribed periods.

In this connection we shall investigate, in the second part, some properties of holomorphic exact (or semi-exact) differential of the form $f \omega$, where $f$ is merely holomorphic and $\omega \in \Gamma_{a}$ (Hilbert space of square integrable differentials). The space $\Gamma_{a}$ has been studied in detail by many authors. A differential $f \omega$ is not square integrable in general provided that $f$ is not bounded. Theorem 2 shows a bilinear relation for our differentials, which reduces, in special case $f \equiv 1$, to our previous results [4], [5]. Theorem 2 will give rise to our main result (Theorem 3 or 4), which gives an information on the growth of holomorphic (or meromorphic) functions expressed as $\varphi / \omega$, where $\omega \in \Gamma_{a}$ and $\varphi$ is exact. 


\section{§I. Abelian differentials with given divisors and periods.}

1. Preliminary. We shall consider an arbitrary open Riemann surface and denote it by R. A Jordan closed curve $\gamma$ on $R$ is called a dividing curve (cycle) if $\gamma$ divides $R$ into two disjoint parts. A dividing cycle is homologous to a sum of oriented boundary contours of a bordered surface containing it.

A region $D$ on $R$ is called a canonical region if (1) $D$ is relatively compact, (2) $D$ and $R-\bar{D}$ have a common boundary which consists of a finite number of analytic ${ }^{1)}$ dividing curves, (3) all components of $R-\bar{D}$ are non-compact. On $R$ there exists a canonical exhaustion $\left\{R_{n}\right\}_{n=1,2, \ldots}$ of $R$ such that $R_{n+1} \supset \bar{R}_{n}$ (closure of $R_{n}$ ) and every $R_{n}$ is a canonical region. Every component of $R_{n+1}-\bar{R}_{n}$ has obviously properties (1), (2).

Let $\Xi=\left\{A_{i}, B_{i} ; C_{j},(i, j=1,2, \ldots)\right\}$ denote a canonical homology basis on $R$, that is, (i) as to the intersection number

$$
A_{i} \times B_{j}=\delta_{i j}, \quad A_{i} \times A_{j}=B_{i} \times B_{j}=0
$$

(ii) $C_{j}$ are the dividing curves, (iii) every cycle on $R$ is (uniquely) homologous to a (finite) linear combination of cycles in $E$. Since $C_{i} \times A_{j}=C_{i} \times B_{j}=0$, every dividing cycle is homologous to a sum of finite number of $C_{j}$.

Let $\left\{R_{n}\right\}$ be a canonical exhaustion of $R$. Then a canonical homology basis $\Xi$ is called a canonical homology basis with respect to $\left\{R_{n}\right\}$ if it has further the following properties; 1) all $A_{i}$ and $B_{i}$ are disjoint with $\left.\bigcup_{n=1}^{\infty} \partial R_{n}, 2\right)$ every $C_{j}$ is a boundary contour of some $R_{n}$, 3) $\Xi \cap \bar{R}_{n}$ forms a canonical homology basis on $\bar{R}_{n}$.

Such a canonical homology basis always exists.

Note that the number of $C_{j}$ contained in every component $\Delta$ of $\bar{R}_{n+1}-\bar{R}_{n}\left(n=1,2, \ldots ; R_{0}=\phi\right)$ is less one than the number of components of $\partial R_{n+1} \cap \Delta$.

1) In the following it is enough to be smooth. 
2. A divisor on $R$ is a locally finite zero dimensional chain on $R$, i.e. $\sum_{i} m_{i} P_{i}$ where the points $P_{i}$ do not cluster in $R$. According to a well-known theorem of Behnke-Stein, for given divisor $\delta$ on $R$ there exists a single-valued meromorphic (or holomorphic) function on $R$ whose divisor is exactly equal to $\delta$. It follows that there exists an Abelian differential on $R$ having a given divisor $\delta_{0}$. Indeed, take an Abelian differential $\omega(\neq 0)$ and let $\delta$ be the divisor of $\omega$, then $f(\omega$ is the required, where $f$ is a meromorphic function with divisor $\delta_{0}-\delta$.

Recently R.C. Gunning and R. Narasimhan [3] proved the existence of a holomorphic exact differential whose divisor is zero. Now we shall show a generalization of this result, that is,

Theorem 1. Let $R$ be any open Riemann surface and $\delta$ be $a$ given divisor on $R$. Then there exists a meromorphic (or holomorphic) differential $\omega$ such that (1) the divisor of $\omega$ is exactly $\delta$ (2) $\omega$ has the prescribed periods along every cycle ${ }^{1)}$ of the canonical homology basis with respect to a canonical exhaustion of $R$.

First of all we shall prove a lemma. Take a canonical exhaustion $\left\{R_{n}\right\}$ and a canonical homology basis $E$ with respect to $\left\{R_{n}\right\}$. For a moment we fix $n$ and write $E=R_{n-1}, F=R_{n}$ and denote by $\left\{\gamma_{1}, \ldots, \gamma_{q}\right\}$, $\left\{\gamma_{q+1}, \ldots, \gamma_{s}\right\}$ the subsets of $\Xi$ contained in $\bar{E}$ and $\bar{F}-\bar{E}$ respectively.

Lemma 1. Let $\omega$ be an Abelian differential on $\bar{F}$. For given $\varepsilon>0$ and complex numbers $\left\{a_{i}\right\}(i=q+1, \ldots, s)$, there exists a holomorphic function $w(p)$ on $R$ such that $|w(p)|<\varepsilon$ on $\bar{E}$ and $\widetilde{\omega}=e^{w(p)} \omega$ has the periods;

$$
\int_{\gamma_{i}} \tilde{\omega}= \begin{cases}\int_{\gamma_{i}} \omega, & i=1, \ldots, q \\ a_{i}, & i=q+1, \ldots, s .\end{cases}
$$

Proof. We proceed by following the ingenious method due to $R$.

1) We should choose, of course, the cycles so as to avoid the points with negative coefficients in $\delta$. 
C. Gunning and R. Narasimhan. One can easily find the complex-valued continuous functions $u_{i}(i=1, \ldots, s)$ on $\cup \gamma_{i}$ satisfying the following properties; the supports of $u_{i}$ are mutually disjoint and contained in $\gamma_{i}$, and

$$
\begin{gathered}
\int_{\gamma_{i}} u_{i} \omega \neq 0, \quad(i=1, \ldots, q) \\
\int_{\gamma_{j}} \omega e^{u_{i}}=a_{j}, \int_{\gamma_{j}} u_{j} \omega e^{u_{j}} \neq 0, \quad(j=q+1, \ldots, s) .
\end{gathered}
$$

For $z=\left(z_{1}, \ldots, z_{s}\right) \in C^{s}$ put

$$
\varphi_{i}(z)=\int_{\gamma_{i}} \omega \exp \sum_{j=1}^{s} z_{j} u_{j}, \quad(i=1, \ldots, s)
$$

Then for $a=(\underbrace{0, \ldots, 0}_{q}, \underbrace{1, \ldots, 1}_{s-q})$ we have

$$
\begin{aligned}
& \varphi_{i}(a)=\left\{\begin{array}{l}
\int_{\gamma_{i}} \omega \quad \text { for } 1 \leq i \leq q \\
\int_{\gamma_{i}} \omega e^{u_{i}}=a_{i} \quad \text { for } q+1 \leq i \leq s
\end{array}\right. \\
& \frac{\partial \varphi_{i}}{\partial z_{i}}(a)=\left\{\begin{array}{lr}
\int_{\gamma_{i}} u_{i} \omega \neq 0 & \text { for } 1 \leq i \leq q \\
\int_{\gamma_{i}} u_{i} \omega e^{u_{i}} \neq 0 & \text { for } q+1 \leq i \leq s
\end{array}\right. \\
& \frac{\partial \varphi_{i}}{\partial z_{j}}(a)=\int_{\gamma_{i}} u_{j} \omega e^{u_{i}}=0 \quad \text { for } i \neq j
\end{aligned}
$$

Let $\varphi(z)=\left(\varphi_{1}(z), \ldots, \varphi_{s}(z)\right)$ be a holomorphic map of $\boldsymbol{C}^{s}$ into itself, then

$$
\varphi(a)=\left(\int_{\gamma_{1}} \omega, \ldots, \int_{\gamma_{q}} \omega, a_{q+1}, \ldots, a_{s}\right)
$$

and its Jacobian matrix $\frac{\partial \varphi}{\partial z}(a)$ is non-singular. Let $L=\gamma_{1} \cup \cdots \cup \gamma_{q}$, 
then by the Mergelyan-Bishop's theorem [2] [7], we know that for each $i(1 \leq i \leq q)$ there exist holomorphic functions $w_{i}^{\mu_{i}}$ on $R$ which approximate $u_{i}$ uniformly on $L$ as $\mu_{i} \rightarrow \infty$. For sufficiently large $\mu=\left(\mu_{1}, \ldots, \mu_{q}\right)$ we set

$$
\begin{gathered}
\phi_{i}(z)=\int_{\gamma_{i}} \omega \exp \left(\sum_{j=1}^{q} z_{j} w_{j}^{\mu_{j}}+\sum_{j=q+1}^{s} z_{j} u_{j}\right), \quad(i=1, \ldots, s) \\
\phi(z)=\left(\phi_{1}(z), \ldots, \phi_{s}(z)\right)
\end{gathered}
$$

then $\psi(a)=\varphi(a)$ and the Jacobian matrix $\frac{\partial \psi}{\partial z}(a)$ is non-singular. Let $K=\bar{E} \cup \gamma_{q+1} \cup \cdots \cup \gamma_{s}$, then again by Mergelyan-Bishop's theorem there exist holomorphic functions $w_{i}^{\nu_{i}}$ on $R$ such that $w_{i}^{\nu_{i}} \rightarrow \tilde{u}_{i}\left(\tilde{u}_{i}=u_{i}\right.$ on $\gamma_{i},=0$ on $\bar{E}$ ) uniformly on $K$ as $\nu_{i} \rightarrow \infty$, in particular, $w_{i}^{\nu_{i}} \rightarrow 0$ uniformly on $\bar{E}$. For sufficiently large $\nu=\left(\nu_{q+1}, \ldots, \nu_{s}\right)$ we put

$$
\begin{gathered}
\psi_{i}^{\nu}(z)=\int_{\gamma_{i}} \omega \exp \left(\sum_{j=1}^{q} z_{j} w_{j}^{\mu_{j}}+\sum_{j=q+1}^{s} z_{j} w_{j}^{\nu}\right) \\
\phi^{\nu}(z)=\left(\psi_{1}^{\nu}(z), \ldots, \psi_{s}^{\nu}(z)\right)
\end{gathered}
$$

then each $\psi_{i}^{\nu}(z)$ is an entire function of $z$ and converges uniformly to $\psi_{i}(z)$ on every compact set in $\boldsymbol{C}^{s}$ as $\nu \rightarrow \infty$. Thus, for any $\delta>0$ and sufficiently large $\nu$ there is a point $z^{0}=\left(z_{1}^{0}, \ldots, z_{s}^{0}\right)$ with $\left|z^{0}-a\right|<\delta$ such that $\psi^{\nu}\left(z^{0}\right)=\psi(a)=\varphi(a)$. Hence for given $\varepsilon>0$, if we choose $\delta$ sufficiently small and put

$$
w(p)=\sum_{j=1}^{q} z_{j}^{0} w_{j}^{\mu_{j}}(p)+\sum_{j=q+1}^{s} z_{j}^{0} w_{j}^{\nu}(p)
$$

it follows that $|w(p)|<\varepsilon$ on $\bar{E}$, and the differential $\widetilde{\omega}=e^{w} \omega$ satisfies the condition (1),

q.e.d.

Proof of Theorem 1. Take an Abelian differential $\omega_{0}$ on $R$ whose divisor is exactly equal to $\delta$. We may suppose that $R_{1}$ is a parameter disk. Let $w_{1} \equiv 0$ and $\omega_{1}=\omega_{0} e^{w_{1}}=\omega_{0}$. Suppose we have successively chosen holomorphic functions $w_{1}, \ldots, w_{n-1}$ such that $\left|w_{k}(p)\right|<1 / 2^{k}$ on 
$\bar{R}_{k-1}(k=2, \ldots, n-1)$ and $\omega_{k}=\omega_{0} \exp \left(w_{1}+\cdots+w_{k}\right)$ have the prescribed periods;

$$
\int_{\gamma_{i}} \omega_{k}=\alpha_{i} \quad \text { for every } \gamma_{i} \in \Xi_{k}
$$

where $\Xi_{k}$ is the subset of $\Xi$ contained in $\bar{R}_{k}$ and $\left\{\alpha_{i}\right\}$ are the given complex numbers. Then by Lemma 1 there exists a holomorphic function $w_{n}(p)$ on $R$ such that $\left|w_{n}(p)\right|<1 / 2^{n}$ on $\bar{R}_{n-1}$ and $\omega_{n}$ $=\omega_{0} \exp \left(w_{1}+\cdots+w_{n}\right)=\omega_{n-1} \exp w_{n}$ satisfies the conditions

$$
\int_{\gamma_{i}} \omega_{n}= \begin{cases}\int_{\gamma_{i}} \omega_{n-1}=\alpha_{i}, & \gamma_{i} \in \Xi_{n-1} \\ \alpha_{i}, & \gamma_{i} \in \Xi_{n}-\Xi_{n-1} .\end{cases}
$$

Thus $w(p)=\sum_{k=1}^{\infty} w_{k}(p)$ converges uniformly on every compact set on $R$, hence $w$ is holomorphic on $R$ and it is seen that

$$
\omega=\omega_{0} e^{w}
$$

has the prescribed periods.

COROllary 1. There exists a holomorphic exact differential without zeros on $R$.

Corollary 2. Every Abelian differential $\omega$ on $R$ can be written in the form

$$
\omega=f \omega_{0} \quad \text { or } \quad \omega_{0}=g \omega
$$

where $f$ and $g=1 / f$ are holomorphic functions without zeros on $R$ and $\omega_{0}$ is a differential having the prescribed periods.

\section{§II. A class of holomorphic differentials.}

3. The Hilbert space $\Gamma_{a}$ of square integrable analytic differentials has been studied in detail by many authors. In the connection with 
the results in $\S \mathrm{I}$ we shall here investigate some properties of analytic (exact or semi-exact) differentials on $R$ which are expressed in the form

$$
\varphi=f \omega, \quad \text { where } f \in A(R) \text { and } \omega \in \Gamma_{a}
$$

where $A(R)$ denotes the space of holomorphic functions on $R$. This turns out to give us also some informations on the growth of holomorphic (or meromorphic) functions on $R$ which can be written as

$$
f=\frac{\varphi}{\omega}
$$

Evidently a differential of the form (2) is not square integrable in general, and $\varphi \in \Gamma_{a}$ provided that $f$ is bounded. Functions of the form (3) with $\varphi, \omega \in \Gamma_{a}$ were investigated by L. Myrberg [8] in connection with the value distribution.

4. For our purpose we introduce a coordinate on $R$. Take a canonical exhaustion $\left\{R_{n}\right\}$ of $R$ and mutually disjoint annuli $D_{n}^{i}$ $\left(i=1, \ldots, m_{n}\right)$ each of which includes exactly one contour of $\partial R_{n}$. Let $D_{n}=\bigcup_{i=1}^{m_{n}} D_{n}^{i}$ and assume that $D_{n}(n=1,2, \ldots)$ are disjoint each other. We denote by $\nu_{n}^{i}\left(\right.$ resp. $\left.\nu_{n}\right)$ the harmonic modulus of $D_{n}^{i}$ (resp. $D_{n}$ ), namely, for instance, $\nu_{n}$ is defined by $2 \pi / d_{n}$ where $d_{n}$ is the flux $\int_{C_{n}} * d u_{n}\left(C_{n}=\partial D_{n} \cap R_{n}\right)$ of the harmonic function $u_{n}$ on $D_{n}$ which is $=0$ on $C_{n}$ and $=1$ on $\partial D_{n}-C_{n}$. It follows that $\sum_{i} 1 / \nu_{n}^{i}=1 / \nu_{n}$. Define a function $u$ on $\bigcup_{n=1}^{\infty} D_{n}$ such that

$$
u=\sum_{i=1}^{n-1} \nu_{i}+\nu_{n} u_{n} \quad \text { on } D_{n}(n=1,2, \ldots)
$$

then $u+i v(v ;$ conjugate of $u)$ maps $\bigcup_{n=1}^{\infty} D_{n}$ conformally onto a strip domain : $0<u<R^{\prime}=\sum_{n=1}^{\infty} \nu_{n}(\leq \infty), 0<v<2 \pi$.

5. For any two differentials $\varphi_{j}=f_{j} \omega_{j}(j=1,2)$ with $f_{j} \in A(R)$ 
and $\omega_{j} \in \Gamma_{a}$ consider the integral

$$
L(r)=L\left(r, \varphi_{1}, \varphi_{2}\right)=\sum_{i=1}^{m} \int_{\gamma_{r}^{i}}\left|\varphi_{1}\right| \int_{\gamma_{r}^{i}}\left|\varphi_{2}\right|, \quad 0 \leq r<R^{\prime}
$$

where for $r$ belonging to the interval $I_{n}=\left[\sum_{i=1}^{n-1} \nu_{i}, \sum_{i=1}^{n} \nu_{i}\right], m=m_{n}$ and $r_{r}^{i}$ denote the level curves $\{p \in R ; u(p)=r\}$ contained in $D_{n}^{i}$. Put

$$
L_{n}=L_{n}\left(\varphi_{1}, \varphi_{2}\right)=\min _{r \in I_{n}} L(r) .
$$

Lemma 2. Let $\varphi_{j}=f_{j} \omega_{j}(j=1,2)$ where $f_{j} \in A(R)$ and $\omega_{j} \in \Gamma_{a}$, and $M_{n}^{i}\left(f_{j}\right)=\max _{p \in \bar{D}_{n}^{i}}\left|f_{j}(p)\right|$. If the series

$$
\sum_{n=1}^{\infty} \min _{1 \leq i \leq m_{n}} \frac{\nu_{n}^{i}}{M_{n}^{i}\left(f_{1}\right) M_{n}^{i}\left(f_{2}\right)}
$$

is divergent, then we have

$$
\lim _{n \rightarrow \infty} L_{n}\left(\varphi_{1}, \varphi_{2}\right)=0
$$

Proof. Let $\omega_{j}=a_{j} d u+b_{j} d v$, then by Schwarz's inequality

$$
\begin{aligned}
\int_{\gamma_{r}^{i}}\left|\varphi_{1}\right| \int_{\gamma_{r}^{i}}\left|\varphi_{2}\right| & =\int_{\gamma_{r}^{i}}\left|f_{1}\right|\left|b_{1}\right| d v \cdot \int_{\gamma_{r}^{i}}\left|f_{2}\right|\left|b_{2}\right| d v \\
& \leq \prod_{j=1,2}\left(\int_{\gamma_{r}^{i}}\left|f_{j}\right|^{2} d v \cdot \int_{\gamma_{r}^{i}}\left|b_{j}\right|^{2} d v\right)^{1 / 2} \\
& \leq 2 \pi \frac{\nu_{n}}{\nu_{n}^{i}} \prod_{j=1,2} M_{n}^{i}\left(f_{j}\right)\left(\int_{\gamma_{r}^{i}}\left|b_{j}\right|^{2} d v\right)^{1 / 2} .
\end{aligned}
$$

Summing up from $i=1$ to $m_{n}$ it follows that

$$
L_{n} \leq L(r) \leq 2 \pi \nu_{n} \max \left(\frac{M_{n}^{i}\left(f_{1}\right) M_{n}^{i}\left(f_{2}\right)}{\nu_{n}^{i}}\right)\left(\int_{0}^{2 \pi}\left|b_{1}\right|^{2} d v \cdot \int_{0}^{2 \pi}\left|b_{2}\right|^{2} d v\right)^{1 / 2}
$$

By integrating with respect to $r \in I_{n}$ we have 


$$
\begin{aligned}
L_{n}\left[2 \pi \max _{i} \frac{M_{n}^{i}\left(f_{1}\right) M_{n}^{i}\left(f_{2}\right)}{\nu_{n}^{i}}\right]^{-1} & \leq\left(\iint_{D_{n}}\left|b_{1}\right|^{2} d v \iint_{D n}\left|b_{2}\right|^{2} d v\right)^{1 / 2} \\
& \leq\left\|\omega_{1}\right\|_{D_{n}}\left\|\omega_{2}\right\|_{D_{n}}
\end{aligned}
$$

therefore

$$
\sum_{n=1}^{\infty} L_{n} \min _{i} \frac{\nu_{n}^{i}}{M_{n}^{i}\left(f_{1}\right) M_{n}^{i}\left(f_{2}\right)} \leq 2 \pi\left\|\omega_{1}\right\|\left\|\omega_{2}\right\|<\infty
$$

which implies (7).

Theorem 2. Let $\varphi_{j}=f_{j} \omega_{j}\left(f_{j} \in A(R), \omega_{j} \in \Gamma_{a}, j=1,2\right)$. Suppose $\varphi_{j}$ are semi-exact, and the series (6) is divergent, then there exists a canonical exhaustion $\left\{R_{n_{\nu}}^{\prime}\right\}$ of $R$ and the canonical homology basis with respect to $\left\{R_{n_{\nu}}^{\prime}\right\}$, for which the following type of bilinear relation holds;

$$
\lim _{n_{\nu} \rightarrow \infty}\left[\left(\varphi_{1}, \varphi_{2}^{*}\right)_{R_{n_{\nu}}^{\prime}}-\sum_{A_{k}, B_{k} \subset R_{n_{\nu}}^{\prime}}\left(\int_{A_{k}} \varphi_{1} \int_{B_{k}} \bar{\varphi}_{2}-\int_{B_{k}} \varphi_{1} \int_{A_{k}} \bar{\varphi}_{2}\right)\right]=0
$$

where

$$
\left(\varphi_{1}, \varphi_{2}^{*}\right)_{R_{n_{\nu}}^{\prime}}=i \int_{R_{n_{\nu}}^{\prime}} \varphi_{1} \bar{\varphi}_{2}^{*}=-\int_{R_{n_{\nu}}^{\prime}} \varphi_{1} \bar{\varphi}_{2}
$$

Proof. By Lemma 2 there is a sequence $\left\{\gamma_{n_{\nu}}\right\}$ of the level curves of $u$ such that $L_{n_{\nu}}\left(\varphi_{1}, \varphi_{2}\right) \rightarrow 0\left(n_{\nu} \rightarrow \infty\right)$. Let $R_{n_{\nu}}^{\prime}$ be a canonical region bounded by $\gamma_{n_{\nu}}$. We may suppose that $R_{n_{\nu}}^{\prime}$ and $R_{n_{\nu}}$ have the same canonical homology basis $A_{1}, B_{1}, \ldots\left(\bmod \gamma_{n_{\nu}}\right)$. Now by Green's formula we have

$$
\left(\varphi_{1}, \varphi_{2}^{*}\right)_{R_{n_{\nu}}^{\prime}}=\sum_{A_{k}, B_{k} \subset R_{n_{\nu}}^{\prime}}\left(\int_{A_{k}} \varphi_{1} \int_{B_{k}} \bar{\varphi}_{2}-\int_{B_{k}} \varphi_{1} \int_{A_{k}} \bar{\varphi}_{2}\right)-\int_{\gamma_{n_{\nu}}} \Phi_{1} \bar{\varphi}_{2}
$$

where $\Phi_{1}$ is an integral of $\varphi_{1}$ defined separately on each contour $\gamma_{n_{\nu}}^{i}$ of $\gamma_{n_{\nu}}$. On account of the semi-exactness of $\varphi_{1}, \Phi_{1}$ is single-valued and moreover

$$
\left|\int_{\gamma_{n_{\nu}}^{i}} \Phi_{1} \bar{\varphi}_{2}\right| \leq \int_{\gamma_{n_{\nu}}^{i}}\left|\varphi_{1}\right| \int_{\gamma_{n_{\nu}}^{i}}\left|\varphi_{2}\right|
$$


Adding with respect to $i$ we have $\left|\int_{\gamma_{n_{\nu}}} \Phi_{1} \bar{\varphi}_{2}\right| \leq L_{n_{\nu}}$, and hence our conclusion.

Remark. 1) Theorem 2 is also valid for meromorphic $f_{j}$, provided that the poles of $f_{j}$ fall in $R-\bigcup_{n=1}^{\infty} D_{n}$.

2) If $\omega_{j} \in \Gamma_{\text {ase }}$ and $f_{j} \equiv 1$, Theorem 2 is reduced to the bilinear relation in Kobori-Sainouchi [4] and Kusunoki [5].

Theorem 3. Suppose that a holomorphic differential $\varphi=f \omega(\neq 0)$ is exact on $R$, where $f \in A(R)$ and $\omega \in \Gamma_{a}$ then for any canonical exhaustion $\left\{R_{n}\right\}$ the series

$$
\sum_{i=1}^{\infty} \min _{1 \leq i \leq m_{n}} \frac{\nu_{n}^{i}}{M_{n}^{i}(f)^{2}}
$$

is always convergent, where $M_{n}^{i}(f)=\max _{p \in \bar{D}_{n}^{i}}|f(p)|$.

Note that Theorem 1 tells us that for given $\omega$ there is always such a holomorphic exact differential $\varphi=f \omega$.

Theorem 3 is a direct consequence of Theorem 2, indeed, if the series (9) is divergent, then by (8) we have $\|\varphi\|=0$, hence $\varphi \equiv 0$, which is a contradiction.

More generally, we have the following theorem, which gives also a sufficient condition that a semi-exact differential $\varphi=f \omega\left(\omega \in \Gamma_{a}\right)$ should belong to $\Gamma_{a}$.

Theorem 4. Let $\varphi=f \omega(\neq 0)$ with $f \in A(R)$ and $\omega \in \Gamma_{a}$, for which the series (9) is divergent. Let $\int_{A_{k}} \varphi=a_{k}, \int_{B_{k}} \varphi=b_{k}(k=1,2, \ldots)$. Suppose that $\varphi$ is semi-exact and the series $\sum_{k=1}^{\infty} \operatorname{Im} a_{k} \bar{b}_{k}$ is absolutely convergent, then $\varphi$ belongs to $\Gamma_{a}$ and actually we have

$$
\|\varphi\|^{2}=2 \sum_{k=1}^{\infty} \operatorname{Im} a_{k} \bar{b}_{k}>0 .
$$


In other words, if $\sum \operatorname{Im} a_{k} \bar{b}_{k}$ is absolutely convergent and non-positive, then the series (9) must converge.

Corollary. If $\varphi=f \omega \neq 0\left(f \in A(R), \omega \in \Gamma_{a}\right)$ is semi-exact and has vanishing $A_{i}$-periods $(i=1,2, \ldots)$, then the series (9) always converges.

The assumption is not vacuous on account of Theorem 1 .

6. Here we shall show some variants of the theorems in the previous section.

A) First of all we shall give another evaluation of the integral (4). For $f \in A(R)$, put $m_{n}(f)=\max _{p \in C_{n}}|f(p)|$ where $C_{n}=\left\{p ; u(p)=\sum_{i=1}^{n-1} \nu_{i}\right\}$ $=\partial D_{n} \cap R_{n}$, and $A_{n}(f)=\iint_{D_{n}}\left|f^{\prime}\right|^{2} d u d v, \nu_{n}^{*}=\min _{1 \leq i \leq m_{n}} \nu_{n}^{i}$.

Lemma $2^{\prime}$ For $\varphi_{j}=f_{j} \omega_{j}$ in Lemma 2, if the series

$$
\sum_{n=1}^{\infty} \frac{\nu_{n} \nu_{n}^{*}}{\prod_{j=1,2}\left(\nu_{n} A_{n}\left(f_{j}\right)+\nu_{n}^{*} m_{n}\left(f_{j}\right)^{2}\right)^{1 / 2}}
$$

is divergent, then $\lim _{\bar{n} \rightarrow \infty} L_{n}\left(\varphi_{1}, \varphi_{2}\right)=0$.

Proof. For $f=U+i V \in A(R)$ we evaluate the integral

$$
\varphi^{2}(r)=\int_{C_{r}}|f|^{2} d v=\int_{C_{r}}\left(U^{2}+V^{2}\right) d v, \quad r \in I_{n}
$$

where $C_{r}=\{p ; u(p)=r\}$. Let $\Omega(r)$ be the relatively compact domain bounded by $C_{r}$ and put $A(r)=\iint_{\Omega(r)}\left|f^{\prime}\right|^{2} d \sigma, d \sigma$ being the areal element. Then

$$
2 \varphi(r) \varphi^{\prime}(r)=2 \int_{C_{r}}\left(U \frac{\partial U}{\partial u}+V \frac{\partial V}{\partial u}\right) d v=4 A(r) .
$$

Integrating from $\tilde{\nu}_{n-1}=\sum_{i=1}^{n-1} \nu_{i}$ to $r\left(\in I_{n}\right)$ we get 


$$
\varphi^{2}(r)=4 \int_{\tilde{\nu}_{n-1}}^{r} A(r) d r+\varphi\left(\tilde{\nu}_{n-1}\right)^{2}
$$

Choose a constant $k$ so that $\int_{\gamma_{r}^{i}}(U-k) d v=0$, and set $\bar{U}=U-k$, then by Wirtinger's inequality

$$
\int_{\gamma_{r}^{i}} \bar{U}^{2} d v \leq \frac{1}{4 \pi^{2}}\left(\int_{\gamma_{r}^{i}} d v\right)^{2} \int_{\gamma_{r}^{i}}\left(\frac{\partial U}{\partial v}\right)^{2} d v=\left(\frac{\nu_{n}}{\nu_{n}^{i}}\right)^{2} \int_{\gamma_{r}^{i}}\left(\frac{\partial U}{\partial v}\right)^{2} d v
$$

so that $\int_{C_{r}} \bar{U}^{2} d v \leq\left(\frac{\nu_{n}}{\nu_{n}^{*}}\right)^{2} \int_{C_{r}}\left(\frac{\partial U}{\partial v}\right)^{2} d v$. Hence

$$
\begin{aligned}
A(r)=\int_{C_{r}} U d V & =\int_{C_{r}} \bar{U} d V \leq\left(\int_{C_{r}} \bar{U}^{2} d v \int_{C_{r}}\left(\frac{\partial V}{\partial v}\right)^{2} d v\right)^{1 / 2} \\
& \leq \frac{\nu_{n}}{2 \nu_{n}^{*}} \int_{C_{r}}\left(\left(\frac{\partial U}{\partial v}\right)^{2}+\left(\frac{\partial V}{\partial v}\right)^{2}\right) d v \\
& =\frac{\nu_{n}}{2 \nu_{n}^{*}} \frac{d A(r)}{d r} .
\end{aligned}
$$

It follows therefore from (11) that

$$
\varphi(r)^{2} \leq 2 \frac{\nu_{n}}{\nu_{n}^{*}} A_{n}(f)+2 \pi m_{n}(f)^{2}
$$

Thus we know the conclusion by the same way as in Lemma 2, q.e.d.

Lemma $2^{\prime}$ obviously leads the variants corresponding to Theorems 2,3 and 4 . For instance,

Theorem $3^{\prime}$ If $\varphi=f \omega(\not \equiv 0)\left(f \in A(R), \omega \in \Gamma_{a}\right)$ is exact, then for any canonical exhaustion the series (9) and

$$
\sum_{n=1}^{\infty} \frac{\nu_{n} \nu_{n}^{*}}{\nu_{n} A_{n}(f)+\nu_{n}^{*} m_{n}(f)^{2}}
$$

are always convergent.

It seems us that both series are not comparable in general. 
B) Here we shall consider a regular exhaustion $\left\{R_{n}\right\}$ of $R$ which is, by definition, a canonical exhaustion without the condition that each component of $\partial R_{n}$ is a dividing curve. Let $R_{n}^{i}\left(i=1, \ldots, m_{n}\right)$ be the components of $R_{n}-\bar{R}_{n-1}$ and $\mu_{n}^{i}$ (resp. $\mu_{n}$ ) be the harmonic modulus of $R_{n}^{i}$ (resp. $R_{n}-\bar{R}_{n-1}$ ). Then with a slight modification we can prove the theorems corresponding to Theorems 2,3 and 4 . For instance,

Theorem $3^{\prime \prime}$ Let $\varphi=f\left(\omega \neq 0\left(f \in A(R), \omega \in \Gamma_{a}\right)\right.$ be exact, then for any regular exhaustion $\left\{R_{n}\right\}$ the series

$$
\sum_{n=1}^{\infty} \frac{\mu_{n}^{*}}{M_{n}(f)^{2}} \quad \text { and } \quad \sum_{n=1}^{\infty} \frac{\mu_{n} \mu_{n}^{*}}{\mu_{n} A_{n}(f)+\mu_{n}^{*} M_{n-1}(f)^{2}}
$$

are always convergent, where $M_{n}(f)=\max _{p \in \partial_{n}}|f(p)|, A_{n}(f)=\iint_{R_{n}-\bar{R}_{n-1}}\left|f^{\prime}\right|^{2} d \sigma$ and $\mu_{n}^{*}=\min _{i} \mu_{n}^{i}\left(\geq \mu_{n}\right)$.

We note that in a particular case where $R$ belongs to the class $\mathrm{O}_{G}$ it is known that there exists a regular exhaustion such that $\mu_{n} \geq k_{n}$ for arbitrarily given sequence $\left\{k_{n}\right\}$ of positive numbers.

7. Finally we note that the divergence of a series like (9) gives an information for the growth of Euler characteristic of our surface $R$. Let $\left\{R_{n}\right\}$ be a regular exhaustion of $R$. Then as in sec. 4 we can introduce a coordinate $w=u+i v$ on $\bigcup_{n=1}^{\infty} R_{n}$ instead of $\bigcup_{n=1}^{\infty} D_{n}$.

Let $\varphi=f \omega\left(f \in A(R), \omega \in \Gamma_{a}\right)$ be a holomorphic differential on $R$. If $\varphi$ does not have zeros on $R$ and if the series

$$
\sum_{n=1}^{\infty} \frac{1}{\tilde{M}_{n}(f)^{2}}, \quad \tilde{M}_{n}(f)=\max _{p \in \bar{R}_{n}}|f(p)|
$$

is divergent, then we have

$$
\int^{s} \chi(r) d r=\infty, S=\sum_{n=1}^{\infty} \mu_{n}
$$

where $\chi(r)$ denotes the Euler characteristic of the relatively compact 
region bounded by $\{u(p)=r\}$.

In fact, by the same way as Lemma 2 we know that if the series (12) diverges, then there exists a sequence $\left\{r_{n}\right\}$ such that $r_{n} \rightarrow S$ and

$$
\int_{u=r_{u}}|\varphi| \rightarrow 0, \quad(n \rightarrow \infty) .
$$

While, by Gauss-Bonnet's theorem (cf. [6] Theorem 2) we have then

$$
-\int_{0}^{r} \chi(r) d r+\mathrm{O}(1)=\frac{1}{2 \pi} \int \log |\psi| d v, \quad \varphi=\psi d w .
$$

The right hand side is less than $\log \left(\frac{1}{2 \pi} \int|\psi| d v\right)=\log \left(\frac{1}{2 \pi} \int|\varphi|\right)$, thus (13) follows from (14).

\section{KYoto UNIVERSITY \\ Kyoto Teghnical University}

\section{References}

[1] Behnke, H. und Stein, K. Entwicklung analytischer Funktionen auf Riemannschen Flächen. Math. Ann. 120 (1949) 430-461.

[2] Bishop, E. Subalgebras of functions on a Riemann surface. Pacific J. Math. 8 (1958) 29-50.

[3] Gunning, R.C. and Narasimhan, R. Immersion of open Riemann surfaces. Math. Ann. 174 (1967) 103-108.

[4] Kobori, A. and Sainouchi, Y. On the Riemann's relations on open Riemann surfaces. J. Math. Kyoto Univ. 2 (1962) 211-223.

[5] Kusunoki, Y. On Riemann's period relations on open Riemann surfaces. Mem. Coll. Sci. Univ. Kyoto Ser. A Math. 30 (1956) 1-22.

[6] Kusunoki, Y. Notes on meromorphic covariants. Ibid. 30 (1957) 243-249.

[7] Mergelyan, S. N. Uniform approximation of functions of a complex variable. Amer. Math. Soc. Translation 101.

[8] Myrberg, L. Über meromorphe Funktionen und Kovarianten auf Riemannschen Flächen. Ann. Acad. Sci. Fenn. Ser. A I. Math. 244 (1957).

[9] Sainouchi, Y. On the analytic semiexact differentials on open Riemann surface. J. Math. Kyoto Univ. 2 (1963) 277-293. 